\title{
The Distribution of Mass in (Disk) Galaxies: Maximal or Not?
}

\author{
Stéphane Courteau ${ }^{1}$ \\ Department for Physics, Engineering Physics and Astrophysics, Queen's University, \\ Kingston, ON K7L 3N6, Canada \\ email: courteau@astro.queensu.ca
}

\begin{abstract}
The relative distribution of matter in galaxies ought to be one of the most definitive predictions of galaxy formation models yet its validation is challenged by numerous observational, theoretical, and operational challenges. All galaxies are believed to be dominated by an invisible matter component in their outskirts. A debate has however been blazing for the last two decades regarding the relative fraction of baryons and dark matter in the inner parts of galaxies: whether galaxies are centrally dominated by baryons ("maximal disk") is of issue. Some of those debates have been misconstrued on account of operational confusion, such as dark matter fractions being measured and compared at different radii. All galaxies are typically baryon-dominated (maximal) at the center and dark-matter dominated (sub-maximal) in their outskirts; for low-mass galaxies $\left(V_{\text {tot }} \lesssim 200 \mathrm{~km} \mathrm{~s}^{-1}\right.$ ), the mass of the dark halo equals the stellar mass at least within 2 disk scale lengths, the transition occurs at larger effective radii for more massive galaxies. An ultimate goal for galaxy structure studies is to achieve accurate data-model comparisons for the relative fractions of baryonic to total matter at any radius.
\end{abstract}

Keywords. galaxies: spiral - galaxies: dark matter - galaxies: formation

\section{Introduction}

Ever since the first speculations about the presence of "dark matter" in galaxies (Kapteyn 1922; Oort 1932), astronomers have endeavored to characterize the fraction of visible to total mass at all radii in galaxiest. Because mass is an integrated quantity which accounts for all the material within a given radius, the question about local gravitating mass is perhaps best posed by considering the ratios of local densities or velocities. In this brief review, we examine the various reports of $\mathcal{F}(R) \equiv V_{\text {baryon }} / V_{\text {tot }}$ in disk galaxies, where $V_{b a r}$ is the inferred circular velocity ascribed to the baryonic material and $V_{\text {tot }}$ is the measured circular velocity at a given radius. $V_{b a r}$ is typically obtained by multiplying a galaxy light profile, $L(R)$, by a suitable mass-to-light ratio, $\left(M_{*} / L\right)(R)$. Any difference between $V_{\text {baryon }}$ and $V_{\text {tot }}$ is viewed as a signature for the presence of dark matter.

How galaxies ultimately arrange their baryonic and non-baryonic matter is the result of numerous complex mechanisms involving their mass accretion history, the depth of the potential well, the initital mass function (which ultimately affects feedback and quenching processes), dynamical friction, dynamical instabilities, and more. Analytical models of galaxy formation strive to predict $\mathcal{F}(\mathcal{R})$ (Dalcanton et al. 1997; Dutton et al. 2007; Mo et al. 2010) with mitigated success in light of the above challenges; numerical simulations suffer additional limitations too.

$\dagger$ Courteau et al. (2014) report in their review that Kapteyn (1922) is the first reference to "dark matter" in the astrophysical literature. 
Can observations provide unique determinations of $\mathcal{F}(\mathcal{R})$ as ideal constraints to galaxy formation models? Not yet, but, after decades of muddled debates and incomplete data, a clear picture seems to be emerging.

\section{Mass Models}

Mass models of disk galaxies, where the observed rotation curve is decomposed into its principal gas, stars, and dark matter components, have historically been embraced as the ideal method to separate baryons and dark matter as a function of radius (Bosma 1978; Carignan \& Freeman 1985; van Albada et al. 1985). The pros and cons of this approach are addressed in the review on "Galaxy Masses" by (Courteau et al. 2014). As that review stresses (see also Dutton et al. 2005), fundamental degeneracies between the disk and dark halo models prevent a unique baryon/dark matter decomposition based on rotation curve data alone. Other information or methods must be considered to break these degeneracies.

Among others, the quantity $V_{\text {bar }}$ is obtained indirectly via a stellar or baryonic mass-tolight ratio $\left(M_{*} / L\right)$ which is itself inferred from stellar population studies or dynamical stability analysis. Current stellar population mass-to-light ratios carry an uncertainty of a factor 2 at best (Conroy 2013; Courteau et al. 2014). The latter uncertainty is unfortunately large enough to encompass a full range of baryon fractions at a given radius. A maximal disk obeys

$$
\mathcal{F} \equiv V_{\text {disk }}\left(R_{\max }\right) / V_{\text {tot }}\left(R_{\max }\right)>0.85
$$

where $V_{\text {disk }}$ is the inferred velocity of the disk (stars and gas), $V_{\text {tot }}$ is the total observed velocity, and $R_{\max }$ is the radius at which $V_{\text {disk }}$ reaches its peak value. Therefore, for most disk galaxy mass models, stellar population M/L ratio uncertainties are such that both maximal and sub-maximal disk solutions are allowed (see Fig. 12 of Courteau et al. 2014).

\section{Other Methods, Same Results?}

To circumvent the loopholes of mass modeling, Courteau \& Rix (1999) observed that the residuals of the velocity-luminosity relation, $\Delta \log (L)$, contrasted against those of the size-luminosity, $\Delta \log R(L)$, relation could provide a tighter constraints on $\mathrm{F}$ measured at the peak of the rotation curve $\nmid$. It can be easily shown that $\Delta \log V(L) / \Delta \log R(L)=-/+$ 0.5 for baryon (-0.5) and dark matter $(+0.5)$ dominated galaxies (Courteau \& Rix 1999; Reyes et al. 2011). Fig. 1 gives an example for such residual estimates measured at $\mathrm{R}=2.2$ disk scale lengths from a typical sample of spiral galaxies where $\Delta \log V(L) / \Delta \log R(L) \simeq$ 0; indeed, all spiral galaxies obey this relation (Courteau \& Rix 1999; Courteau et al. 2007; Dutton et al. 2007; Reyes et al. 2011). The null slope essentially indicates that both baryons and dark matter contribute to the galaxy dynamics at $2.2 R_{d}$. Various galaxy structure models whereby the dark matter is compressed adiabatically by the cooling baryons as the galaxy stabilizes dynamically yield a mapping between $\Delta \log V(L) /$ $\Delta \log R(L)$ and the baryon fraction $\mathcal{F}$. These models yield, on average for bright spiral

$\dagger$ If a disk is assumed to be exponential, its rotation curve will peak at 2.2 disk scale lengths, $R_{d}$. However, only $\sim 20 \%$ of all disk galaxies have near exponential disks (Courteau et al. 2007). Thus the so-called $2.2 R_{d}$ peak in the baryonic rotation curve is an ill-defined metric since most disk galaxies have Freeman Type-II profiles. A more practical fiducial radius that enables the comparison of both late and early-type galaxies in a non-parametric fashion, is the half-light, or effective, radius, $R_{e}$. For an exponential disk, $2.2 R_{d}=1.3 R_{e}$. To avoid additional confusion, $\mathcal{F}(\mathcal{R})$ should use either metric radii (however spoiled by distance errors) or radii relative to $R_{e}$. 


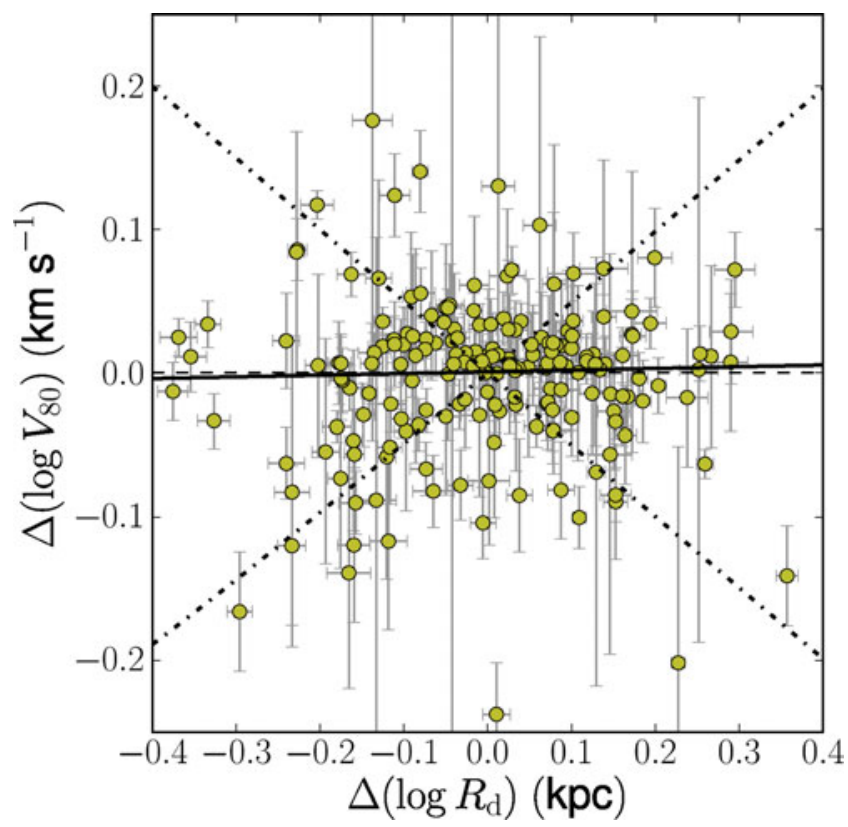

Figure 1. Correlation between velocity residuals $\Delta\left(\log V_{80}\right)$ and disk size offsets $\Delta\left(\log R_{d}\right)$ from a velocity-luminosity and size-luminosity relations for a sample of typical spiral galaxies covering a broad range of surface brightnesses. The best-fit linear relation for $\Delta(\log V(L)) / \Delta(\log R(L))$ has a slope consistent with zero (solid line). Predicted trends for a pure self-gravitating disk model (slope $=-0.5$ ) and a pure NFW DM halo model (slope $=+0.5)$ are also shown (dotdashed lines). Figure taken from Reyes et al. (2011); in agreement with Courteau \& Rix (1999) and Dutton et al.(2007).

galaxies, $\mathcal{F}\left(2.2 R_{d}\right)=0.6-0.72$. The lower value applies if only adiabatic contraction is at play; a higher value is found if other mechanisms (e.g. feedback) oppose the adiabatic contraction (Courteau \& Rix 1999; Dutton et al. 2007). By virtue of Eq. (2.1) above, galaxy disks are thus, on average, sub-maximal at $2.2 R_{d}$. Since mass scale as $V_{\mathrm{tot}}^{2}, \mathrm{M}_{\mathrm{DM}} / \mathrm{M}_{\mathrm{tot}}=50-70 \%$ at $2.2 R_{d}\left(=1.3 R_{e}\right.$ for a pure exponential disks $)$. Baryon/DM equality in disk galaxies is thus roughly achieved at $1 R_{e}$. Reyes et al. (2011) showed that $\mathcal{F}$ is controlled by the surface density of the baryonic material; i.e. if the latter is high, so is $\mathcal{F}$ (see also Fig. 23 in Bovy \& Rix 2013).

A similar result is obtained via the "velocity dispersion" method of (van der Kruit 1988), as applied by many for a full suite of disk galaxies (Bottema 1993), edge-on systems (Kregel et al. 2005), or face-on spiral galaxies Bershady et al. (2011); Martinsson et al. (2013). For a self-gravitating, radially exponential disk with vertical profile of the form $\rho(R, z)=\rho(R, 0) \operatorname{sech}^{2}\left(z / z_{0}\right)$, van der Kruit (1988) and Bottema (1993) showed that the peak circular velocity of the stellar disk, $V_{\text {disk }}$, measured at $R=2.2 R_{\mathrm{d}}$ can be related to the vertical velocity dispersion, $V_{z}$, and the intrinsic thickness (or scale height) of the disk, $z_{0}$, via:

$$
V_{\text {disk }}\left(R_{\text {peak }}\right)=c\left\langle V_{z}^{2}\right\rangle_{R=0}^{1 / 2} \sqrt{\frac{R_{\mathrm{d}}}{z_{0}}} .
$$

where $c \simeq 0.88\left(1-0.28 z_{0} / R_{\mathrm{d}}\right)$ (Bershady et al. 2011). A more detailed discussion of Eq. (3.1) is presented in van der Kruit \& Freeman (2011) (see their Section 3.2.4).

$V_{\text {disk }}\left(R_{\text {peak }}\right)$, the contribution of the baryonic disk to the total velocity where the rotation curve reaches its peak (at $R_{\text {peak }}$ ), can thus be measured directly if the disk scale 
length, $R_{d}$, the scale height, $z_{0}$, and the vertical component of the velocity dispersion, $V_{z}$, are known. For face-on systems, $V_{z}$ and $R_{d}$ can be measured but $z_{0}$ must be inferred; and vice versa for edge-ons. Van der Kruit's method is thus statistical in nature and relies on a number of (potentially noisy) scaling transformations. Bovy \& Rix (2013) also point out that the velocity dispersions measured from integrated light, as in most applications of Eq. (3.1) for external galaxies so far, do not trace the older, dynamically-relaxed stellar populations very well. The younger, brighter, stellar populations traced by the lightweighted spectra tend to have a lower velocity dispersion therefore biasing $V_{\text {disk }}\left(R_{\text {peak }}\right)$ low and thus favoring sub-maximal disks. Sample selection also plays a substantial role (see Fig. 2 below).

The DiskMass project has made use of van der Kruit's formalism to extract $V_{\text {disk }}\left(R_{\text {peak }}\right)$ for a sample of 46 nearly face-on (inclinations $\simeq 30$ degrees) galaxies with rotation velocities between $100 \mathrm{~km} \mathrm{~s}^{-1}$ and $250 \mathrm{kms}^{-1}$ (Bershady et al. 2011). This survey uses integral-field spectroscopy to measure stellar and gas kinematics using the custom-built SparsePak and PPAK instruments. For 30 DiskMass galaxies covering a range of structural properties, Martinsson et al. (2013) report that the fraction $\mathcal{F}$ ranges from 0.45 to 0.85 (the maximum disk threshold) and increases with luminosity, surface brightness, rotation speed, and redder color. The average value of their sample is $\mathcal{F}=0.57 \pm 0.07$, in agreement with Courteau \& Rix (1999).

Similar and broader results have been achieved through very different techniques for disk dominated systems: such as fluid dynamical modeling (Weiner et al. 2001; Kranz et al. 2003; Athanassoula 2014, see the latter reference for a more critical outlook) and strong gravitational lensing (Barnabè et al. 2012; Dutton et al. 2013). For instance, because gravitational lensing is sensitive to projected mass while dynamics trace the mass within ellipsoids, their combination provides additional geometrical dimensions to disentangle the baryons and dark matter in galaxies; this approach is especially efficient for highly-inclined disk galaxies (Dutton 2014). These methods, reviewed in Courteau et al. (2014), are consistent on average with $\mathcal{F}\left(2.2 R_{d}\right)=0.6-0.7$, but also show a broad range of $\mathcal{F}$ as a function of maximum circular velocity or disk size. In their hydrodynamical modeling of five grand design non-barred galaxies, Kranz et al. (2003) presciently suggested that galaxy disks appear to be maximal if $V_{\text {max }}>200 \mathrm{~km} \mathrm{~s}^{-1}$, sub-maximal otherwise. The later summarizes rather well the more complete picture about the mass distribution in galaxies that is now clearly emerging.

Figure 2 is a compilation of dark matter fractions, $f_{D M}=1-\mathcal{F}^{2} \approx\left(V_{D M} / V_{t o t}\right)^{2}$, at $\sim 2.2 R_{d}$ for various galaxy samples discussed above (others are discussed below), as a function of total circular velocity.

The DiskMass survey (blue points) predominently samples small-to-average size galaxies thus avoiding the regime of disk maximality (at $2.2 R_{d}$ ). Issues about additional sensitivity to young versus old stellar populations addressed above may also explain the DiskMass dark matter fractions above the mean model (Dutton et al. 2011). The cyan pentagons represent the parameter space for the Tully-Fisher residual analysis of the Courteau et al. (2007) sample of spiral galaxies with (open pentagon) and without (filled pentagon) adiabatic contraction (Dutton et al. 2007). The Courteau and DiskMass samples target essentially the same sub-maximal galaxy types. Unlike those samples, the SWELLS gravitational lensing survey is intrinsically biased towards high mass, predominently maximal, systems (Barnabè et al. 2012; Dutton et al. 2013). The Milky Way study of Bovy \& Rix (2013) uses a method similar to that of (van der Kruit 1988) but with the added benefit that stellar populations can be disentangled, thus providing less biased measurements of the true underlying structure (scale length, rotation and dispersion) 


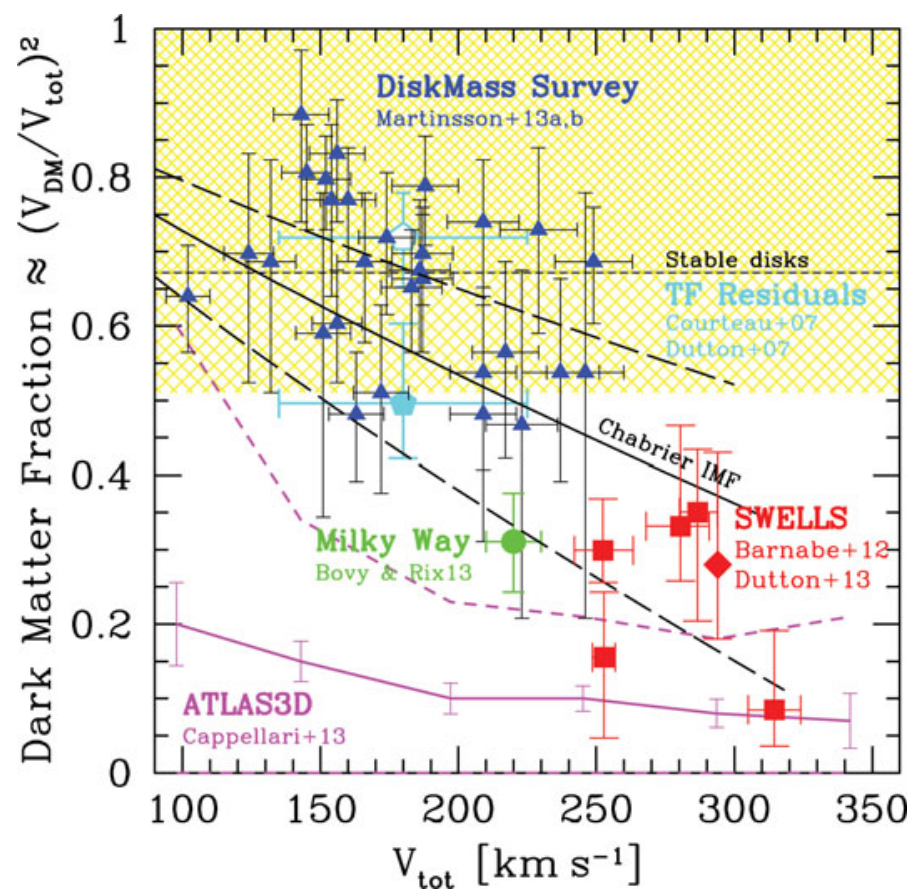

Figure 2. Dark matter fraction at $\sim 2.2 R_{d}$ as a function of total circular velocity for samples discussed in the text. A clear trend is observed such that the dark matter fraction increases with circular speed; indeed below $250 \mathrm{~km} \mathrm{~s}^{-1}$, dark matter dominates $(>50 \%)$ the total mass budget of the galaxy at $2.2 R_{d}$. The yellow shading shows the stability criteria (Efstathiou et al. 1982) for stellar disks $\left(f_{D M} \simeq 0.67\right.$; short-dashed black line and above) and gaseous disks $\left(f_{D M} \simeq 0.51\right)$. The black line represents a model for galaxy scaling relations assuming a Chabrier initial mass function (Dutton et al. 2011); the long-dashed black lines are the $1 \sigma$ dispersion in galaxy sizes. Figure prepared with Aaron Dutton.

of the dynamical tracer. Their analysis results in a low mass-weighted disk scale length, $R_{d}=2.15 \pm 0.14 \mathrm{kpc}$, and a correspondingly low estimate of $f_{D M}=0.31 \pm 0.07$ at $2.2 R_{d} \dagger$.

The short-dash boundary delineates the region where baryon-heavy disk galaxies become disk unstable. The criterion for stability is $V_{\text {circ }}\left(R_{d} / G M_{\text {disk }}\right)^{1 / 2} \gtrsim 1.1$ (Efstathiou et al. 1982) $\ddagger$. The yellow shading shows the stability criteria for stellar $\left(f_{D M}>0.67\right)$ and gaseous $\left(f_{D M}>0.51\right)$ disks.

Figure 2 (left) also shows the distribution of 260 ATLAS3D early-type galaxies (Cappellari et al. 2013), using $V_{\text {circ }}=1.38 \sigma_{8}$ to transform ATLAS3D velocity dispersion estimates, $\sigma_{8}$, to circular velocity $V_{\text {circ }}$ (Dutton et al. 2011). This confirms that ATLAS3D probes ETG dynamics in a regime proper to maximal "baryons".

The baryon-DM equality by mass in disk galaxies is achieved roughly at $1 R_{e}$. While ATLAS3D spectral maps do not extend beyond $1.5 R_{e}$, deeper dynamical studies reaching

$\dagger$ Various investigations of the Milky Way mass density profiles are reported in Table 1 of Courteau et al. (2014). The early studies of Dehnen \& Binney (1998) and Englmaier \& Gerhard (1999) already pointed to a maximal MW disk. Measurements of $\mathcal{F}$ for the Milky Way hinge significantly on estimates of its disk scale length. A high estimate of $R_{d}$ biases $\mathcal{F}$ high.

$\ddagger$ See Foyle et al. (2008) for an alternate, numerical derivation, of this stability criterion as controlled by the ratio of the disc mass fraction, $m_{d}$, to the halo spin parameter, $\lambda$. If $\lambda<m_{d}$, the galaxy will be bar unstable. All galaxy mass models ought to be tested for the stability of their disk against the formation of a central bar (Widrow et al. 2003). 
out to $\sim 4-5 R_{e}$ suggest that the baryon-DM mass equality in ETGs occurs at $\sim 2 R_{e}$ or beyond (Thomas et al. 2007; Deason et al. 2012; Morganti et al. 2013; Oguri et al. 2014), albeit with significant scatter.

\section{Future Prospects}

The trends observed in Figure 2 and the characterization of baryon-DM transitions in galaxies are significant constraints that galaxy formation models have yet to reproduce. This is partly due to additional complications such as the degeneracy between the baryon or dark matter fractions, the stellar initial mass function, adiabatic contraction, and various counter-acting non-gravitational effects (e.g. feedback, dynamical friction) (Dutton et al. 2011; Trujillo-Gomez et al. 2011; Oguri et al. 2014). The advent of new extensive dynamical models of galaxies based on wide-field integral field surveys, such as CALIFA, MaNGA, SAMI (out to $\sim 2 R_{e}$ ) and SLUGGS (reaching $\sim 6-10 R_{e}$ ) heralds a promising future for mapping the mass distributions at all radii in galaxies. Model depenednces and biases could at least be partially offset with reduced sampling errors.

Let us no longer ask if galaxies are maximal or not (they all are!); let us simply decompose accurately the mass profiles of galaxies into their major components (e.g. gas, stars, and dark matter, or bar, bulge, disks, and halos) from the center out to the largest galactocentric radii, and using as many dynamical tracers as possible, in order to derive a reliable continuum of dynamical properties across the Hubble sequence and over time.

\section{Acknowledgements}

I acknowledge support from the Natural Science and Engineering Research Council of Canada for funding via a generous Research Discovery Grant. Figure 2 was prepared with Aaron Dutton. Aaron Romanowsky suggested valuable references too.

\section{References}

Cappellari, M., McDermid, R. M., Alatalo, K., et al. 2013, MNRAS, 432, 1862

Athanassoula, E. 2014, MNRAS, 438, L81

Barnabè, M., Dutton, A. A., Marshall, P. J., et al. 2012, MNRAS, 423, 1073

Bershady, M. A., Martinsson, T. P. K., Verheijen, M. A. W., et al. 2011, ApJ, 739, LL47

Bosma, A. 1978, "The distribution and kinematics of neutral hydrogen in spiral galaxies of various morphological types," Ph.D. thesis (University of Groningen).

Bottema, R. 1993, A\&\&A, 275, 16

Bovy, J. \& Rix, H.-W. 2013, ApJ, 779, 115

Carignan, C. \& Freeman, K. C. 1985, ApJ, 294, 494

Conroy, C. 2013, ARAËA, 51, 393

Courteau, S., Cappellari, M., de Jong, R. S., et al. 2014, Reviews of Modern Physics, 86, 47

Courteau, S., Dutton, A. A., van den Bosch, F. C., et al. 2007, ApJ, 671, 203

Courteau, S. \& Rix, H.-W. 1999, ApJ, 513, 561

Dalcanton, J. J., Spergel, D. N., \& Summers, F. J. 1997, ApJ, 482, 659

Deason, A. J., Belokurov, V., Evans, N. W., \& McCarthy, I. G. 2012, ApJ, 748, 2

Dehnen, W. \& Binney, J. 1998, MNRAS, 294, 429

Dutton, A. A., in "Galaxy Masses as Constraints of Formation Models", Proceedings IAU Symposium No. 311, 2014 M. Cappellari \& S. Courteau, eds.

Dutton, A. A., Treu, T., Brewer, B. J., et al. 2013, MNRAS, 428, 3183

Dutton, A. A., Conroy, C., van den Bosch, F. C., et al. 2011, MNRAS, 416, 322

Dutton, A. A., van den Bosch, F. C., Dekel, A., \& Courteau, S. 2007, ApJ, 654, 27 
Dutton, A. A., Courteau, S., de Jong, R., \& Carignan, C. 2005, ApJ, 619, 218

Efstathiou, G., Lake, G., \& Negroponte, J. 1982, MNRAS, 199, 1069

Englmaier, P. \& Gerhard, O. 1999, MNRAS, 304, 512

Foyle, K., Courteau, S., \& Thacker, R. J. 2008, MNRAS, 386, 1821

Kapteyn, J. C. 1922, ApJ, 55, 302

Kranz, T., Slyz, A., \& Rix, H.-W. 2003, ApJ, 586, 143

Kregel, M., van der Kruit, P. C., \& Freeman, K. C. 2005, MNRAS, 358, 503

Martinsson, T. P. K., Verheijen, M. A. W., Westfall, K. B., et al. 2013, A 6 A, 557, AA131

Mo, H., van den Bosch, F. C., \& White, S. 2010, Galaxy Formation and Evolution, by Houjun Mo, Frank van den Bosch , Simon White, Cambridge, UK: Cambridge University Press, 2010 ,

Morganti, L., Gerhard, O., Coccato, L., Martinez-Valpuesta, I., \& Arnaboldi, M. 2013, MNRAS, 431,3570

Oguri, M., Rusu, C. E., \& Falco, E. E. 2014, MNRAS, 439, 2494

Oort, J. H. 1932, Bull. Astron. Inst. Neth., 6, 249

Reyes, R., Mandelbaum, R., Gunn, J. E., Pizagno, J., \& Lackner, C. N. 2011, MNRAS, 417, 2347

Thomas, J., Saglia, R. P., Bender, R., et al. 2007, MNRAS, 382, 657

Trujillo-Gomez, S., Klypin, A., Primack, J., \& Romanowsky, A. J. 2011, ApJ, 742, 16

van Albada, T. S., Bahcall, J. N., Begeman, K., \& Sancisi, R. 1985, ApJ, 295, 305

van der Kruit, P. C. \& Freeman, K. C. 2011, ARA\&A, 49, 301

van der Kruit, P. C. 1988, A\&A, 192, 117

Weiner, B. J., Sellwood, J. A., \& Williams, T. B. 2001, ApJ, 546, 931

Widrow, L. M., Perrett, K. M., \& Suyu, S. H. 2003, ApJ, 588, 311 